\title{
Diagnostic Testing of Oil-impregnated Paper Insulation in Pro- rated Power Transformers under Accelerated Stress
}

\author{
M. K. Pradhan \\ T. S. Ramu \\ Department of High Voltage Engineering \\ Indian Institute of Science, Bangalore (INDIA) \\ E-mail: manoj@hve. iisc.ernet.in
}

\begin{abstract}
In the recent past, a considerable amount of work has been published on the dynamics of aging of oil impregnated paper (OIP) insulation in transformers. The insulation is always subjected to stresses of different types at the same point in time. Among the more important stress are, electrical, thermal and their combination. Insulating properties of both solid (cellulose paper) and liquid (mineral insulating oil) phases deteriorate with time due to an electro-thermal process called aging. The status of insulation at a given point in time can therefore be assessed by monitoring such of those properties of insulation which are sensitive to the amount of aging, so as to be able to take and appropriate corrective measures to avoid / postpone impending insulation failure. This procedure, called Diagnostic Testing and Condition Monitoring (DTCM) aims to avoid catastrophic failure.
\end{abstract}

The aim of the present investigation is to try and determine the most sensitive parameters which can serve as indices of aging and hence as a reasonably accurate indicator of possible imminent failure of apparatus insulation in service. To this end, carefully controlled laboratory aging experiments have been performed on about eight nominally identical and scaleddown models of pro-rated units incorporating all the essential features of actual power transformers under normal operating electric stress and accelerated thermal stress. The paper details the DTCM program designed to acquire aging data.

\section{INTRODUCTION}

Mineral oil impregnated paper (OIP) is the insulation of choice in transformers and more often this system deteriorates rapidly at elevated electrical and thermal stresses occurring singly and in combination. The degradation is of the two constituent phases of insulation, viz; the cellulose and mineral insulating oil, due to an irreversible thermo chemical reaction rate process, the byproducts of the reaction among others are, carbon oxides, and a class of hydrocarbons including what are called furans. These compounds exist in both a free state as well as in a dissolved state. Accurate detection and quantification of these by-products seems to offer a possible method of identifying the nature and severity of the faults in the transformer. The status of the oil could be assessed relatively easily, by direct sampling and physico-chemical analysis. The cellulose part, on the other hand, is more difficult to sample and analyze.

A perusal of current literature shows that, various properties of insulation such as Gas generation (GG), evolution of furans, Degree of Polymerization (DP) of cellulose, Dielectric Strength of both oil and paper, Partial Discharge (PD) parameters, dissipation factor and its tip-up, change in capacitance $(\mathrm{C})$, infestation of parasitic particles, moisture content, different spectroscopic parameters and the like can be used as indices of the quantum of deterioration in both the solid and the liquid phase.

To monitor the changes in the values of several properties, a series of invasive, minimally invasive and non-invasive or diagnostic tests are instituted. While DP and Dielectric strength measurements on cellulose are invasive in nature, DGA, measurement of PD and dielectric parameters form the diagnostic tests. It is generally known that the status of insulation is more definitely indicated by the destructive tests, but is often precluded. Qualitative information, although less accurate are however preferred for reasons well known.

The DP being a structural property, in as much as that it gives an account of the number of monomer units in cellulose, establishment of a correlation or otherwise with an associable diagnostic property would serve to improve the accuracy of depiction of the quality of insulation at any given point in time. For example, thermo-chemical degradation of cellulose derivatives evolve a class of furans which remain in a dissolved state in oil as well as be present in gas phase in the Bucholtz relay chamber and which are concomitant with reduction of DP. A quantification of the furans using appropriate detection techniques would indirectly point to the fact that the DP has changed, and considerably at that.

The present work is an exercise in identifying the most sensitive parameters, which could serve as reasonably accurate indices of aging of the transformer insulation. To this end, nominally identical pro-rated and scaled-down models incorporating all the essential features of actual power transformers under normal operating conditions have been fabricated and tested at elevated thermal stresses, electric stress being maintained at normal levels. Depending upon the degradation data acquired from this, the aging experiment, a possible monitoring schedule worked out.

\section{Philosophy of Accelerated Aging}

Aging experiments under operating stress conditions in laboratory environment require, inordinately long times before credible failure data can be acquired. This implies a considerable expenditure in terms of time and costs of running aging experiments. A calculated saving thereon can be effected by taking recourse to the fact that, phenomenologically, the time to failure of insulation follows an inverse power function of electrical stress and, under thermal stress, it is an Arrhenius rate process. It is possible, under certain restrictions, to work out a method of acquiring 
failure data in a much shorter time by judiciously increasing the stresses applied during the aging experiments. There are certain rules for working out the acceleration factor for the applied stresses. For the sake of brevity, the more important of them are included here.

- The mechanism of aging shall remain invariant with stress

- The accelerating variable shall not exceed a factor of about $20 \%$ of its normal level.

- Under thermal stress, the life seems to be in conformity with the Montsinger rule of half-life.

- The ratio of the experimental duration to the expected life shall not be less than about 0.05 .

The nature of the stress used for laboratory experiment could be either continuous in time or, stepped at appropriate levels. It has been assumed that the thermal stress is such that the laws of first order reaction kinetics are not violated. In the present work, an IEEE loading pattern has been used [1], [2].

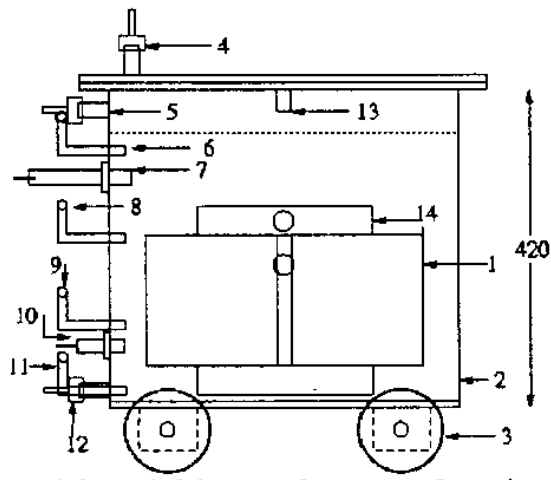

Figure 1 - A Schematic Diagram of prototype, front view

Table I: Legend to Fig.1

\begin{tabular}{|l|l|l|l|}
\hline Legend & Label & Legend & Label \\
\hline 1 & Winding & 8 & MOT $^{*}$ probe-1 \\
\hline 2 & Tank & 9 & MOT $^{7}$ probe-1 \\
\hline 3 & Wheel & 10 & LV bushing \\
\hline 4 & Online gas valve & 11 & BOT probe \\
\hline 5 & Nitrogen valve & 12 & Bottom drain valve \\
\hline 6 & TOT probe & 13 & Eyelet \\
\hline 7 & HV bushing & 14 & Frame \\
\hline
\end{tabular}

\section{EXPERIMENTAL}

The genesis of the IEEE loading pattern is the (EAP). Scale down modes of actual power transformers were designed in the authors' laboratory and fabricated by a local transformer manufacturer. Fig.1 shows a detailed drawing of the test object to scale. Eight $5 \mathrm{kVA}, 220 \mathrm{~V} / 5 \mathrm{kV}, 50 \mathrm{~Hz} 1$-phase, core type, $O N$, nitrogen cushion transformers have been designed. Mineral oil impregnated electrical grade Kraft paper and Manila paper have been used for winding insulation and end insulation respectively. Before impregnation with oil, the paper was dried under vacuum to have moisture content of less than $0.5 \%$.
Each of the specimens has been provided with three valves with septum arrangement (one at the top cover, other two at top and bottom of the front cover) for sampling and filling gas as well as oil. Sampling arrangement has been provided on the top cover for paper insulation also. Provisions have been made to measure the top, middle and bottom oil temperature of the model using thermo-couples

\section{Aging Process}

Before conducting the aging experiment a proper loading schedule was decided depending upon the electrical and thermal stress required for paper as well as oil for degradation. The stepped stress loading cycle used in the experiment is shown in the Fig.2. It can be seen that there are three blocks in a cycle, representing three different current loading and hence temperature. The time duration of these blocks are adjusted such that the amount of aging during each blocks remain nearly the same.

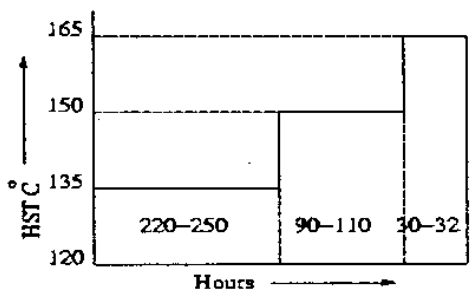

Figure 2 - A typical Loading cycle

Temperature mentioned here is calculated HST [3] of each loading phase and the duration of exposure at each temperature in hours has been selected such a way that it would require 6-10 cycles to reach the average time to endof-life for a transformer model.

In addition to the routine tests, insulation resistance (IR), Capacitance between HV-to-LV and HV-to-tank and dissipation factor, performed on all the specimens, partial discharge (PD) measurements, DGA Furan and DP measurements were also carried out.

\section{Condition Monitoring of The Specimen}

In a high voltage high power transformer, a careful consideration of the two phases, winding insulation (solid part), and the insulating (and cooling) liquid medium are to be considered as separate entities. Therefore, the specimen has been monitored during the load cycle (on-line) and after each load cycle a series of invasive and non-invasive diagnostics tests were performed on both of the phases. More often than not, insulation failure is due mainly to thermal stress and electrical stress acts as a promoter of the chemical reactions in both media. Monitoring techniques are complimentary [4], [5].

\section{Monitoring of Liquid Phase}

Top oil, middle oil and bottom oil temperature of the specimen have been continuously monitored during load cycle. The most important tests carried out on oil phase after 
the end of each load cycle, are; moisture content, capacitance and dissipation factor (Tan $\delta$ ), dielectric breakdown strength, micro-particle infestation, detection, measurement and quantification of the dissolved gases.

Monitoring of liquid phase has the potential to assess the condition of solid phase as the by-product of degradation of paper (carbon oxides and furans) are carried to the liquid phase and remain there as dissolved gases.

\section{Monitoring of Solid phase}

The hot spots occur almost always in the winding of transformer caused often by transient and/or deliberate over loading. It is generally not possible to directly measure the hot spot temperature. Hot spot temperature is estimated using thermal model with the help of top oil temperature and load current. Except furan analysis there is no non-invasive test to assess the condition of the paper insulation After the end of each cycle paper samples were taken out to perform both DP and break-down strength measurements.

\section{RESULTS AND DISCUSSION}

In keeping with the concepts in accelerated thermal stress aging, a series' of experiments have been designed and performed in a planned manner. A number of properties have been identified as being reasonably sensitive indices of degradation and monitored continuously. For the sake of brevity, only a few of the more important of them are included here.

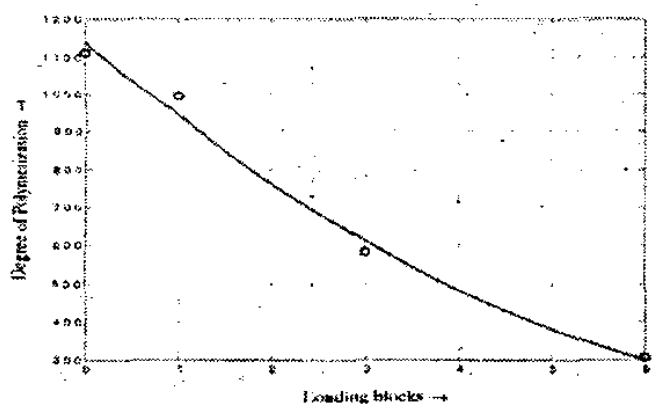

Figure 3 - Degree of polymerization vs aging time

The changes in the DP vs. duration of aging with the applied load cycle shown in Fig. 3. It may be observed that the DP shows, a decreasing trend over successive load / time block. In fact, at the end of the 6th block, the DP can be seen to have come down to a value of 310 , which is already approaching the lower limit for acceptance.

The BDV of paper sampled from end insulations has been measured using Rogowski electrodes with diameter of 40 $\mathrm{mm}$ (ground) and $10 \mathrm{~mm}$ (HV), shown in Fig.4. The BDV of oil has been measured after every cycle using a spheresphere, $(10 \mathrm{~mm}$ each), geometry with a separation of 3.3 $\mathrm{mm}$. It can be seen from Fig.5 that, the BDV of oil is sensitive to the amount of aging in that it exhibits a nearly monotonically decreasing pattern.

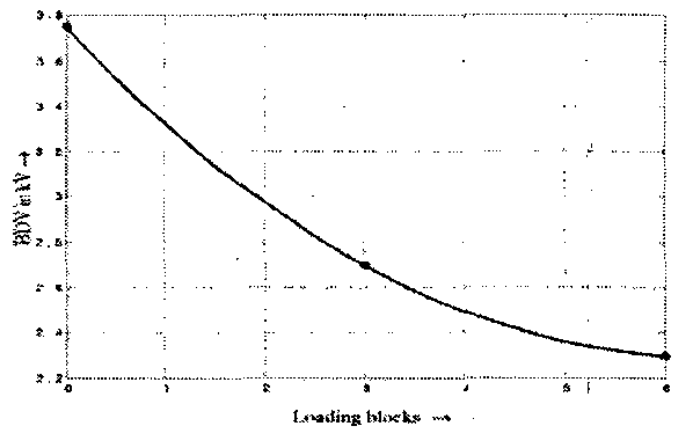

Figure 4 - Breakdown Voltage of creep paper

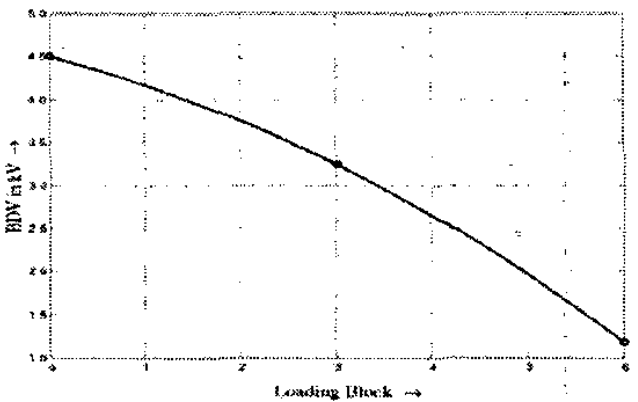

Figure 5 - Breakdown Voltage of oil

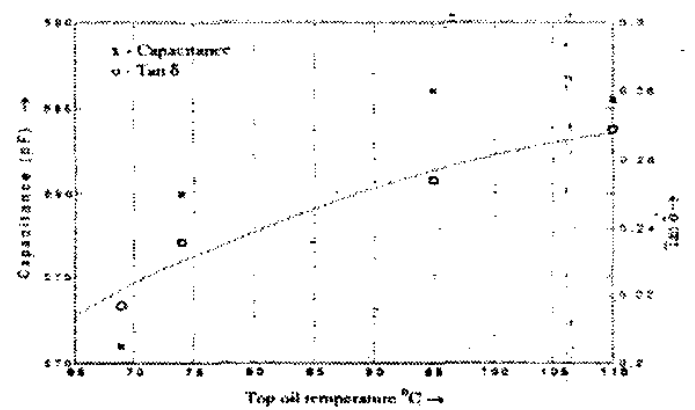

Figure 6 - Capacitance and dissipation factor vs TOT

Capacitance and dissipation factor were measured using a Tettex Bridge (model-2805 HA) as a function of estimated temperature, and results shown in Fig.6. In Fig.7, the changes in Tan $\delta$ with respect to the duration of aging have been depicted. The results are in near conformity. with the phenomenological concepts.

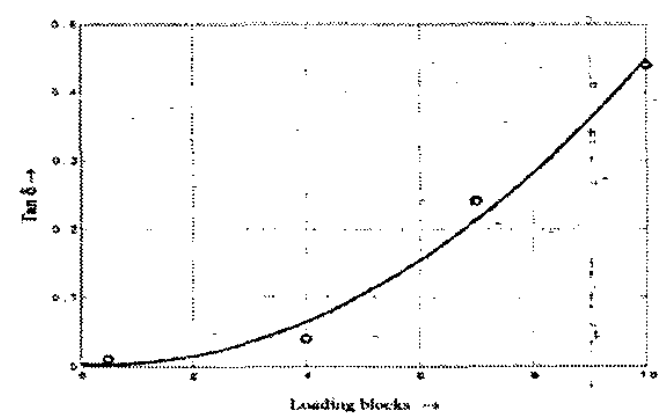

Figure 7 - Capacitance and dissipation factor Aging 
In the course of insulation aging, free particles are generated and infestation of the particles also serves as a qualitative measure of degradation. The reason for the breakdown strength to be lowered with aging duration is generally traced to the increase in the size and number density of particles and hence it was decided to obtain information on this aspect. Fig.8, is a bar plot of the particle count and it can be seen that the particle count is sensitive to aging but it is not always possible to make conclusive statements in this regard. The quantity shown here is the total count, ranging in particle size from $2.5 \mu \mathrm{m}$ to $100 \mu \mathrm{m}$.

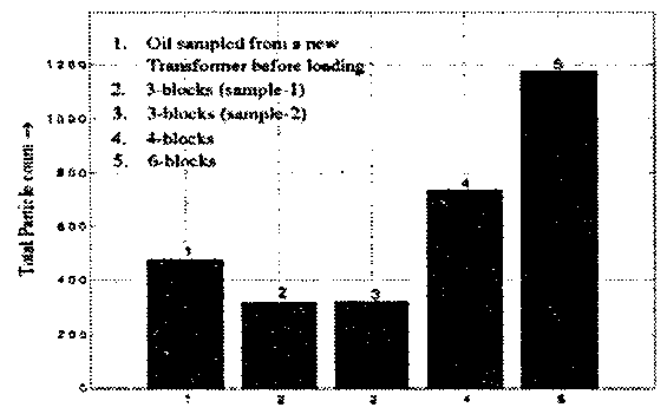

Figure 8 - $\mu$-particle count

Table II: Comparison of sensitivity of the parameters

\begin{tabular}{|c|c|c|c|}
\hline $\begin{array}{c}\text { Parameter of } \\
\text { choice }\end{array}$ & $\begin{array}{c}\text { Sensitivity } \\
\% \text { per block }\end{array}$ & Monotnicity & $\begin{array}{c}\text { Qualitative } \\
\text { Assessment }\end{array}$ \\
\hline Particle count & 10.1 & Non-monotonic & Good \\
\hline Tan $\delta$ & 7.5 & Monotonic & Very good \\
\hline BDV oil & 13.0 & Monotonic & Good \\
\hline BDV paper & 6.4 & Monotonic & Good \\
\hline DP & 12.1 & Monotonic & Excellent \\
\hline
\end{tabular}

In Table II, the qualification and efficacy of above parameters, viability of their use from the stated viewpoint of their sensitivity (measured in terms of the quantum of change in the property per unit aging duration) to thermal aging and monotonicity in their changes as a function of duration of aging have been presented. It can be seen that, the qualitative grading of Tan $\delta$ seems to be considerably higher than others. The particle count and BDV of oil as indices of degradation because of its non-monotonic characteristic. Also, it can be pointed here is that such a qualitative qualification of the parameters will be useful in developing realistic reliability estimation system based on fuzzy logic methods.

The work covered here has precedence in the IEEE and IEC loading guides. The load intensities have been worked out based on these documents. Authors' own experience [3] in thermal modeling of power transformer insulation has been utilized to a great measure. Given that the prorated units are not nun to failure, that some kind of censoring on time has been adopted, it becomes necessary to enunciate a criterion for failure. The serviceability or end-of-life of power transformers in actual service conditions is based on system reliability and economic considerations. However, the end point criterion for pro-rated transformer units under laboratory controlled accelerated thermal stress can be made out more effectively as tests of invasive nature can be performed as and when they are deemed fit.

Due to financial and time constraints in running long duration experiments, a serious need was felt to economize on these factors. Several criteria for terminating the aging experiments cited. In order to be able to scientifically justify the censoring methodology for the aging experiments, recourse to IEEE proof test strategy [1], [2] on its merits.

In conclusion, it can be pointed out that, an improved thermal aging experimental design has been instituted so as to obtain on-line information by DTCM has been suggested. The results of a series of fault finding experiments performed to assess the condition of insulation at different points in time suggested here can be seen to be powerful enough to apply to actual insitu tests on power transformers in service.

\section{REFERENCES}

[1] IEEE Std C57.100-1999, "IEEE standard test procedure for thermal evaluation of Liquid-immersed Distribution and Power Transformers".

[2] IEEE Std 259-1999, "IEEE standard test procedure for evaluation of systems of insulation for dry-type specialty and general-purpose transformers".

[3] M K Pradhan and T S Ramu, "Estimation of the Hottest Spot Temperature (HST) in Power Transformers Considering Thermal Inhomogeniety of the Windings", IEEE Trans. Power Delivery (in press).

[4] IEEE Guide for Aging Mechanisms and Diagnostics in Evaluating Electrical Insulation Systems, ANSU/IEEE Std $943-1986$, Nov 15,1985

[5] IEEE Guide for Acceptance and Maintenance of Insulating Oil in Equipment, IEEE Std C57.106-2002, 8 Nov 2002 